\title{
Smart Collectibles: Unlocking The Value of Non-Fungible Tokens (NFTs)
}

\author{
Andrew Fai \\ afai@destation.org
}

\begin{abstract}
NFT-based art is at the forefront of a new wave of digital ownership that enables creators to retain control of their work and create and sell ownership rights for their digital media. But digital art is just the tip of the iceberg: this same technology can unlock access to any unique physical or digital resource, including products, services, and event access.

We call these NFTs - used to access and determine ownership of resources off the blockchain - 'Smart Collectibles.' Smart Collectibles have applications that range from digital gaming and media to finance and property. They are also a key enabler of Web $3.0^{1}$ and the Metaverse, an always-on shared digital universe where people can work, play, and hang out.

By creating this document, we aim to inspire and encourage creators, communities, and businesses to continue to innovate in this space. With Smart Collectibles, artists and organizations can enable access to value in a way that is traceable and highly secure and even stack access to multiple forms of value onto the primary non-fungible tokens themselves.
\end{abstract}

\section{Introduction}

The way we create, store, and trade value is changing. NFT-based art - such as the $\$ 69$ million Beeple collection sold at Christie's ${ }^{2}$, the $\$ 590,000$ Nyan Cat NFT ${ }^{3}$, and Jack Dorsey's $\$ 2.9$ million first Tweet $^{4}$ - is at the forefront of a new wave of digital ownership that will transform how businesses and individuals create, provide, and access value.

The same blockchain technology used to mint $^{5}$ and trade NFT-based art can be used to create NFTs $^{6}$ that act as unique digital keys'. These NFT keys, called 'Smart Collectibles,' can unlock access to almost anything - including services, experiences, and digital or physical products. Because Smart Collectibles are stored on the blockchain and held in a digital wallet, they provide creators and owners more control over the use of their data and goods.

\footnotetext{
${ }^{1}$ Web 3.0 is the next generation of internet services and will shift ownership of data from centralized corporations back into the hands of individual users. We'll cover this topic in more detail later in the paper.

${ }^{2} \mathrm{https}$ // www.christies.com/features/Monumental-collage-by-Beeple-is-first-purely-digital-artwork-NFT-to-com e-to-auction-11510-7.aspx

${ }^{3}$ https://www.coindesk.com/nyan-cat-nft-ethereum-meme

${ }^{4}$ https://venturebeat-com.cdn.ampproject.org/c/s/venturebeat.com/2021/05/20/setting-the-record-straight-onnfts-the-most-misunderstood-financial-advancement-in-history/amp/

${ }^{5}$ Minting is the process used to create an NFT on the blockchain.

${ }^{6}$ Ethereum.org, Non-fungible Tokens (NFT), https://ethereum.org/en/nft/

${ }^{7}$ We've used a comparison with keys as an analogy for how Smart Collectibles enable access to data. This should not be confused with the concept of public and private keys which are used in the secure encryption and decryption of data held on the blockchain.
} 


\section{What Are Smart Collectibles?}

We define Smart Collectibles as NFTs that unlock access to unique value held off the blockchain.

In the simplest sense, a Smart Collectible is a unique piece of code you hold in a digital wallet and use as an access token for something of value. As NFTs, the Smart Collectible code is created on the blockchain ${ }^{8}$, which means each Smart Collectible is unique, secure, and easy to sell or transfer to another user.

The unique access could refer to a physical or digital item, experience, or service. In fact, anything that someone might sell could be termed 'value.' The value is referred to as unique because having a Smart Collectible in your digital wallet enables you to access something that others cannot.

When we say the value is held off the blockchain, all this means is that you're using the Smart Collectible to get access to something that is not coded within the Smart Collectible's metadata, such as a digital download or physical event. Access to these can be assigned exclusively to specific Smart Collectible holders by using the tokenID, an identifier that is unique to every NFT.

Because providing this access only needs this unique identifier, and not ownership of the Smart Collectible itself, any person or organization can map new access to value to any Smart Collectible. The Smart Collectible itself (the file held in your wallet) is unchanged. New value can be mapped for exclusive access by the Smart Collectible owner any time after the original NFT has been created.

Because they unlock value, Smart Collectibles invite comparisons with physical keys and digital passwords, but they can do much more. In comparison to these, Smart Collectibles have many improvements and benefits: they are more secure, have many more uses, can each be used to enable access to multiple pieces of value, and much more:

A physical key (e.g. a house key) allows you to unlock one specific lock. Typically this means you can access value in one physical location (such as a house). Because the key can be stolen or copied, allowing access to multiple locations increases risk. Additionally, if the value (the house) is sold, security can only be assured by changing the keys and locks, because there is no way of knowing whether a previous owner made a copy of the key or not. Owning the key is not enough to prove ownership of the value, so third-party verification is essential.

A digital password allows you to access one specific digital account (in most cases). The value that that account provides is controlled by the organization that issues it. Passwords can be reused for multiple accounts, but this substantially increases the risk if the password is stolen, guessed, or forgotten. In some cases, an account can be used to access additional value (for example, logging in to another service using your Facebook credentials). Selling is impractical and compromises security (passwords must be changed after the account is sold), and is not typically done.

In comparison to these, Smart Collectibles have many improvements and benefits. Each Smart Collectible has the potential to unlock access to many types of value, and this value can be added without limit by both the creator of the token and others. Because the Smart Collectible is made on the blockchain, it is nearly impossible to tamper with.

Selling a Smart Collectible is easy and secure; NFT standards and marketplaces mean they can be bought and sold freely in a liquid market. Access automatically transfers to the new owner

\footnotetext{
${ }^{8}$ Blockchain is a decentralized digital ledger, or database, technology that tracks transactions. In this paper, we assume that the blockchain refers to the Ethereum blockchain, but any blockchain that supports smart contracts could be used for Smart Collectibles.
} 
after a sale, and because the information is stored on a publicly verifiable ledger (the blockchain), there is no need for third-party verification.

Let's take a look at the difference. See Figure 1.

\begin{tabular}{|l|l|l|l|}
\hline & Physical Key & Digital Password & Smart Collectible \\
\hline Accessing Value & One physical location & $\begin{array}{l}\text { One or more digital } \\
\text { accounts }\end{array}$ & $\begin{array}{l}\text { Physical \& digital } \\
\text { value (no limit) }\end{array}$ \\
\hline Value Creation & $\begin{array}{l}\text { Can fit and open one } \\
\text { or more locks }\end{array}$ & $\begin{array}{l}\text { Value typically only } \\
\text { added by the } \\
\text { company that issues } \\
\text { the accounts }\end{array}$ & $\begin{array}{l}\text { Value can be added } \\
\text { without limit by both } \\
\text { the creator and } \\
\text { others }\end{array}$ \\
\hline Security & $\begin{array}{l}\text { Can be stolen or } \\
\text { copied }\end{array}$ & $\begin{array}{l}\text { Can be stolen, } \\
\text { guessed, or forgotten }\end{array}$ & $\begin{array}{l}\text { Nearly impossible to } \\
\text { tamper with }\end{array}$ \\
\hline Selling & $\begin{array}{l}\text { Selling compromises } \\
\text { security (key/locks } \\
\text { must be changed } \\
\text { after the sale) }\end{array}$ & $\begin{array}{l}\text { Selling compromises } \\
\text { security (password } \\
\text { must be changed } \\
\text { after account sold) }\end{array}$ & $\begin{array}{l}\text { Selling on liquid } \\
\text { markets is easy and } \\
\text { secure. Access } \\
\text { automatically } \\
\text { transfers to the new } \\
\text { owner after sale. }\end{array}$ \\
\hline Ownership & $\begin{array}{l}\text { Requires verification } \\
\text { From third-party }\end{array}$ & $\begin{array}{l}\text { Requires verification } \\
\text { From third-party }\end{array}$ & $\begin{array}{l}\text { Ownership } \\
\text { information stored on } \\
\text { the publicly verifiable } \\
\text { ledger (blockchain) }\end{array}$ \\
\hline
\end{tabular}

Figure 1: Comparing Smart Collectibles with Physical Keys and Digital Passwords

\subsection{Use Case: Event Ticketing}

These benefits can be easily explained by looking at a real-life use case. For example, a Smart Collectible could be used as a ticket to a physical event, such as a concert. Let's see how it could work over the lifetime of the Smart Collectible:

Creation: Each unique ticket is a Smart Collectible, which when sold is transferred to the purchaser's digital wallet ${ }^{9}$. The owner of that wallet is then given access to the event by proving their ticket ownership using an NFC-enabled device, like a smartphone. Because the Smart Collectible 'ticket' is held on the blockchain, it is nearly impossible to tamper with it ${ }^{10}$.

If the owner chooses to resell the ticket before the event, this can be done simply and securely without the aid of an expensive intermediary. Additionally, the Smart Collectible can be programmed so that part of the transaction fee goes to the creator of the event, ensuring the event benefits from any resales that happen.

Added Value: After the event, the event organizer adds value to the Smart Collectible by enabling the owner to access an exclusive video of the concert. They also allow ticket holders access to exclusive discounts for an online store where they can purchase merchandise related to

\footnotetext{
${ }^{9} \mathrm{~A}$ digital wallet is an app used to store digital tokens and cryptocurrency.

${ }^{10}$ It is nearly impossible to steal a Smart Collectible cryptographically, however there is still a vulnerability to social engineering techniques where someone tricks you into sending them the Smart Collectible or to providing access to your Digital Wallet, or by stealing your private keys (if you have stored them somewhere).
} 
the event. The creator can stack any amount of value on the same Smart Collectible. This does not need to be added all at once; creators can continue to add value to a Smart Collectible indefinitely.

Sale: Over time, the band who gave the concert grows in fame, and the Smart Collectible ticket continues to accrue value due to the unique bonuses it enables access to and as a collectible item itself. The holder decides they want to sell it and lists it on a popular NFT marketplace, enabling collectors to bid for it in an auction. After a competitive auction, the ticket and its associated benefits sell as a collector's item for the equivalent of $\$ 1,000$. The band that issued the Smart Collectible receives a percentage of the sale, in this case, 10\%. They will continue to receive this every time the Smart Collectible is sold, enabling them to continue to benefit from the value they provide.

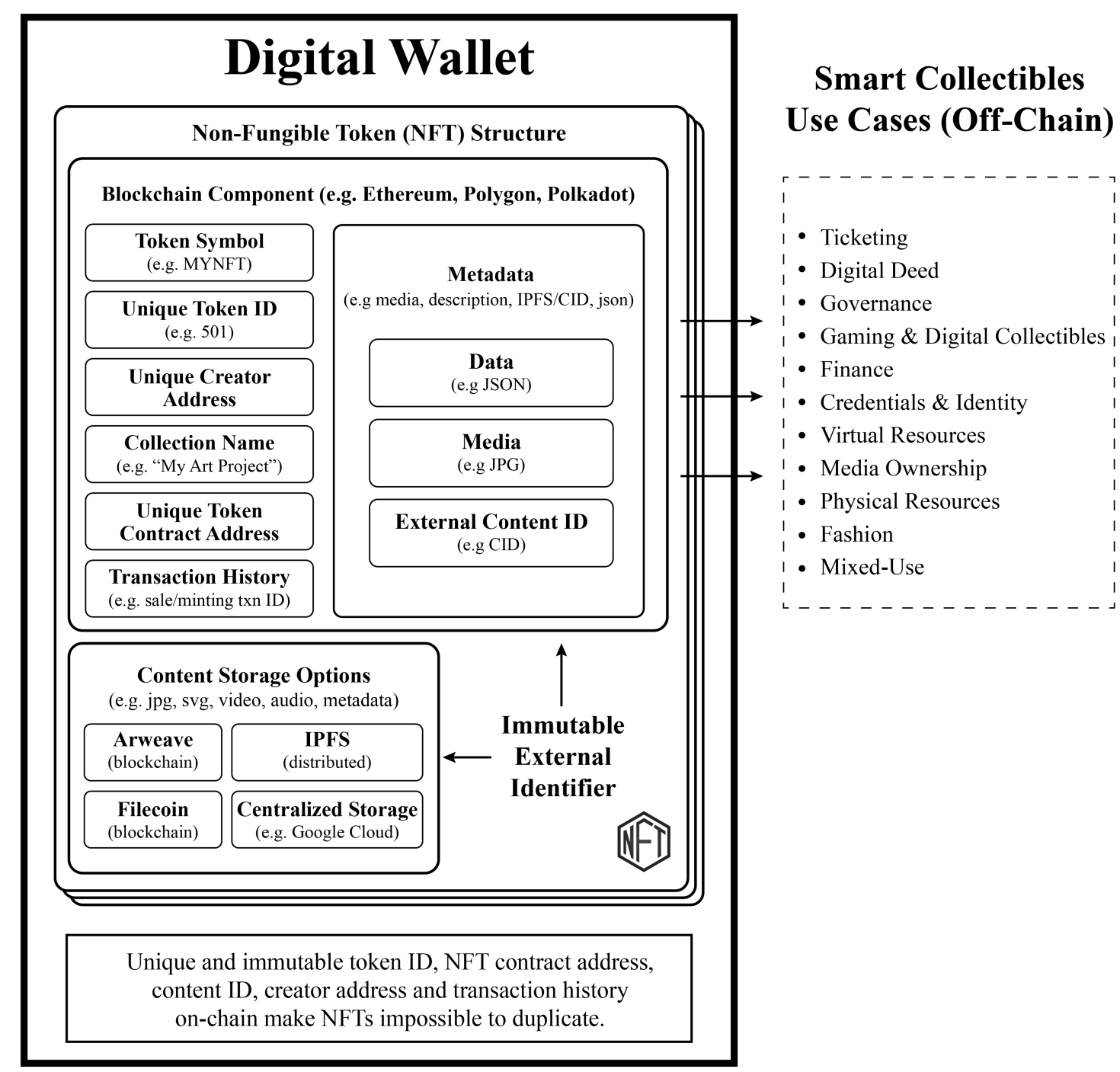

Figure 2: Unlocking Off-Chain Functionality With Smart Collectibles

As you can see from Figure 2, when creators begin to use NFTs to provide access to more value than can be held in metadata, a plethora of functionality is unlocked. When creators make NFTs like this (which we categorize as Smart Collectibles), they access many benefits:

- Create and sell secure digital 'keys' for accessing almost any type of value

- Mix different types of value on the same Smart Collectible, enabling them to create unique 
value propositions tailored to their customer's needs and business model.

- Add additional value after creating it, perhaps months or even years later

- Include code in the metadata so that they automatically receive a percentage of the sale price when the Smart Collectible is resold on a secondary market

- Add value to Smart Collectibles made by other creators

For Smart Collectible owners, this enables them to:

- Securely purchase assets that continue to accrue value

- Benefit from multiple forms of value through just one Smart Collectible

- Easily access the value by having all their Smart Collectibles in their digital wallet without having to remember multiple usernames and passwords

- Securely sell or trade their Smart Collectibles whenever they wish

\section{Smart Collectibles Use Cases}

Smart Collectibles enable creators to limit access to digital or physical items or functionality securely, and as such, have a wide range of use cases. In addition to the ticketing and events example above, use cases are detailed below and illustrated in Figure 3.

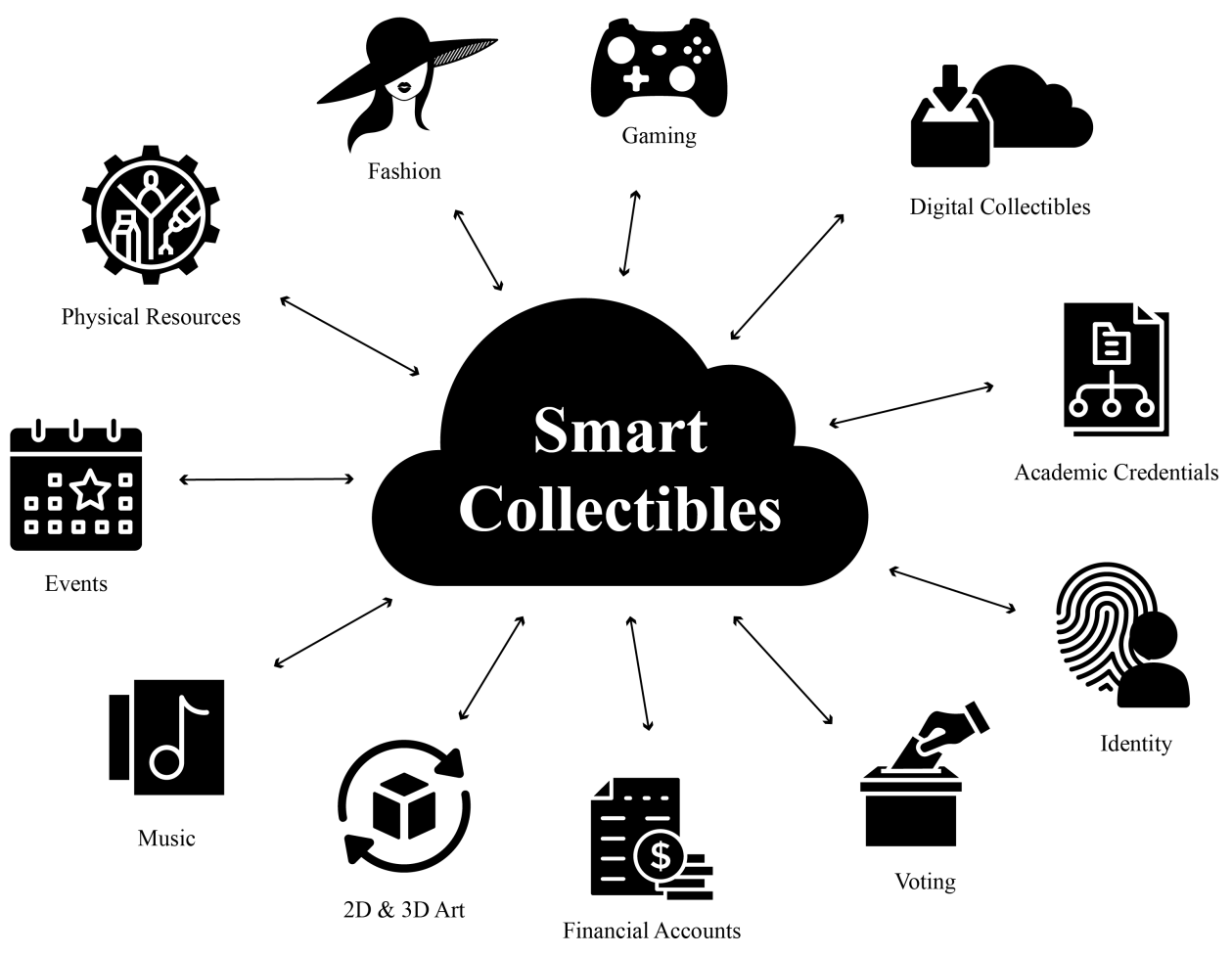

Figure 3: Smart Collectible Use Cases 


\subsection{Gaming \& Digital Collectibles}

Collect, use and sell unique digital collectibles that enable users to access unique gameplay or abilities within one or many games. These include digital Collectible Card Games (CCGs) and unique items and resources (weapons, apparel, items) in online multiplayer games.

A single Smart Collectible could unlock access in one or many games. For example, a publisher may enable one collectible to unlock the same clothing style in multiple games, enabling users to better define their own digital identity.

Additionally, NFTs and Smart Collectibles could also replace user accounts and passwords. In this scenario, selling an account is as easy as trading the Smart Collectible: whoever holds the Smart Collectible can access the account.

This can also be beneficial for publishers, who currently do not benefit when accounts are sold on a secondary market: the Smart Collectible can be coded to reward game publishers with a percentage of the price every time an account is sold on.

\subsection{Finance}

A Smart Collectible can be assigned rights or deeds to dividends, securities, properties, businesses, and more. Furthermore, Smart Collectibles can be used to reassign value. For example, a Smart Collectible can be assigned as a beneficiary in a will, enabling someone to pass on their estate to another person, or multiple persons in the case of multiple NFT owners.

\subsection{Credentials \& Identity}

A Smart Collectible could be used as a unique and secure method of authenticating a person's identity or other credentials and using that information to enable or disable access to specific resources or actions. In this case, the Smart Collectible would not be tradable to prevent identity fraud. Potential uses include voting, identity cards, and access to financial accounts. One project working on this is IdToken ${ }^{11}$.

\subsection{Voting}

NFTs and Smart Collectibles can provide voting rights for owners that enable them to have their say in how a platform, business, community organization, DAO (see below), or even country is run.

Each voting token (smart collectible) enables the owner to take a vote. Depending on the setup, owners may be able to own multiple voting tokens or just one, and tokens may be tradable or non-tradable. In some cases, users may assign their voting rights to another individual to enable them to vote on their behalf (while still retaining ownership of their Smart Collectible).

Many DAOs currently use ERC-20 tokens (cryptocurrencies) as voting tokens, enabling people to vote according to how many tokens they hold and to split their vote if they wish (quadratic voting ${ }^{12}$ ). Smart Collectibles can be used similarly but could also enable other voting options. For example, rarer Smart Collectibles may offer a greater voting weight (more votes) than more common ones.

\footnotetext{
${ }^{11}$ IdToken uses biometric data to create the private key, ensuring users are able to access their data even if they lose their digital wallet. Read more in the whitepaper by Eduardo Talamo and Alma Pennacchi, IdToken: the new decentralized approach to digital identity, https://dblp.org/rec/conf/openidentity/TalamoP20

${ }^{12}$ Quadratic voting allows someone to allocate votes according to how strongly they prefer one option. For example, a person may have 5 votes and allocate 3 to one option and 2 to another, to express a slight preference.
} 


\subsection{Decentralized Autonomous Organizations (DAOs)}

A Decentralized Autonomous Organization (DAO) is a fully-decentralized organization that runs autonomously according to the rules coded into it when created. Instead of having a CEO or board of directors, the DAO is managed by the owners of its associated tokens, which enable them to have voting rights on what actions the organization takes next.

\subsection{Virtual Resources}

Smart Collectibles are an easier-to-use, more secure alternative to passwords. Instead of requiring multiple secure passwords for different websites, users sign in with their digital wallets. This digital wallet can hold one or many Smart Collectibles that enable the user access to their online accounts. Projects such as Sequence and Rainbow Wallet are already making this a reality.

Many online users use their Facebook or Google accounts to achieve a similar result; however, relying on these third-parties creates significant privacy concerns. Smart Collectibles offer a cryptographically-secure alternative that protects the user's privacy.

Additionally, it is easy to sell a Smart Collectible on an NFT marketplace, and doing so does not compromise your other accounts. In contrast, it is not only difficult to price and sell a Google or Facebook account, but by doing so, you are giving the buyer everything that you have used that account for.

\subsection{Media Ownership}

NFT-art is just the tip of the iceberg. Smart Collectibles can be used to provide functionality for owners of art, music, 3d work, and even text-based work. Owners will be able to create virtual galleries of their media for friends to look at.

\subsection{Physical Resources}

Using an NFC-enabled ${ }^{13}$ device, such as a smartphone, Smart Collectibles can enable access to physical resources (and prevent unauthorized users from accessing). For example, a manufacturer may use Smart Collectibles to allow employees to access and use specific machinery. This could be combined with credentials to ensure employees only use equipment they have been trained to use, or other functionality to prevent use outside of work hours.

\subsection{Fashion}

Smart Collectibles would enable high-end fashion retailers and end-users to authenticate genuine products and prevent counterfeiting by linking each unique piece with a Smart Collectible. Users will authenticate each piece, check ownership history, access care guides, and more.

In practice, this is difficult to achieve since it is possible to sell the physical asset without updating the token record. One solution is to provide a centralized authentication service that manages the transferable of ownership, holding the product and fee in escrow until the token record has been updated.

\footnotetext{
${ }^{13} \mathrm{NFC}$, or Near Field Communication, allows electronic devices to exchange information at close range. It is used in contactless cards and when making payments using a smartphone.
} 


\subsection{Mixed-Use}

One of the most significant benefits of using Smart Collectibles is that multiple different use cases can be implemented on a single Smart Collectible. This provides creators, communities, and businesses with nearly unlimited opportunities to build value for their followers and customers.

\section{How Do Smart Collectibles Work?}

Smart Collectibles are a type of token stored on the blockchain. It is the underlying blockchain technology, along with the code that makes up the token, that provides Smart Collectibles with the benefits that set them apart from other ways to access value. Let's break it down:

A blockchain is a decentralized ledger, which means that the transaction history (who owns what) is stored on many nodes ${ }^{14}$. This differs from traditional centralized ledgers, where there is a single copy of the data held on a central node. Because there are many copies, it is nearly impossible to lose or tamper with the data ${ }^{15}$. See Figure 4.

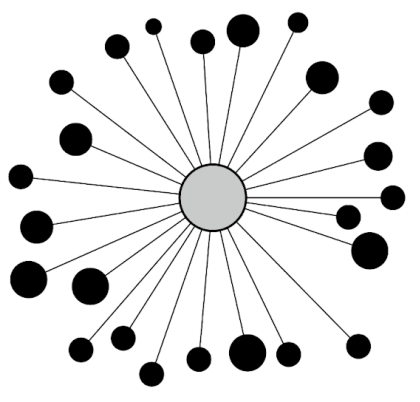

Centralized Network

All the nodes are connected under a single authority

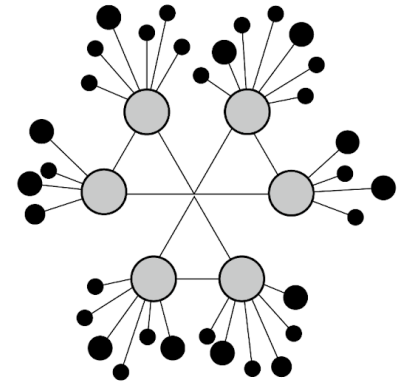

Decentralized Network

No single authority server controls the nodes, they all have individual entity

Figure 4: Centralized and Decentralized Network Models

The Bitcoin blockchain, for example, tracks transactions of Bitcoin, a fungible ${ }^{16}$ digital cryptocurrency that can be used as a store of value and to purchase goods and services ${ }^{17}$. These cryptocurrencies are not Smart Collectibles.

More recently, blockchains such as Ethereum have enabled additional functionality called smart contracts, which are programs that are stored on the blockchain ${ }^{18}$. Among other things, smart

\footnotetext{
${ }^{14}$ Nodes are the computers that make up the infrastructure of the blockchain. Full nodes hold a copy of the blockchain and are used to verify new transactions. Most blockchains have thousands of full nodes, which makes them very secure - a bad actor would need to hack or control $51 \%$ of nodes to be able to make changes.

${ }^{15}$ Shermin Voshmgir, Tokenized Networks: Web3, the Stateful Web, https://blockchainhub.net/web3-decentraliz ed-web/

${ }^{16} \mathrm{~A}$ fungible token is interchangeable from another token of the same type. Both Bitcoins and Dollars are examples of fungible tokens. A single dollar is indistinguishable from another dollar.

${ }^{17}$ Satoshi Nakamoto, Bitcoin: A Peer-to-Peer Electronic Cash System, https://bitcoin.org/bitcoin.pdf

${ }^{18}$ Nick Szabo, Smart Contracts: Building Blocks for Digital Markets, https://www.fon.hum.uva.nl/rob/Courses/ InformationInSpeech/CDROM/Literature/LOTwinterschool2006/szabo.best.vwh.net/smart_contracts_2.html
} 
contracts can be used to create tokens that can be owned and traded.

These tokens are created using set standards that govern how they can be used. Tokens created using the ERC-20 standard are fungible and can be used as currencies, while the ERC-721 19 standard is used to mint Non-Fungible Tokens (NFTs) ${ }^{20}$.

The ERC-721 standard ensures that every NFT has functionality such as transferability and that every NFT is unique. The uniqueness of the NFT enables the creation of Smart Collectibles: each NFT has a unique tokenID that a decentralized app (dApp) ${ }^{21}$ can use as an input to unlock access to any type of value. A newer standard, ERC- $1155^{22}$, can be used to create both fungibles, like currencies, and non-fungibles, like NFTs and Smart Collectibles.

\subsection{Use Case: Digital Sword}

For example, imagine a unique digital sword created as an NFT on the Ethereum blockchain using the ERC-721 standard. Its unique tokenID means only one user can own this sword at a time.

If that sword does nothing else, apart from existing, then it is an NFT, but not a Smart Collectible. This does not mean it is worthless, of course: if the artist is in high demand, this NFT could be bought or sold for significant sums, just like a physical piece of art. However, if its tokenID enables access to further value held off the blockchain we now classify the NFT as a Smart Collectible.

Now, imagine the same digital sword, but now when you hold that token in your digital wallet, you can equip and wield it in a popular online game, unlocking new abilities and powers you did not previously have access to. To achieve this, you log in with your digital wallet so that the game can check the tokenIDs of the tokens in your wallets. The game can be programmed to enable different functionality for certain tokenIDs.

This functionality will continue to be available to the owner as long as they hold the Smart Collectible in their wallet. If they transfer or sell the Smart Collectible to another person, the access to the unique value is transferred and is no longer available to the seller.

\subsection{Smart Collectibles vs. NFTs}

All Smart Collectibles are NFTs, and because of this, they have the same properties as NFTs they are non-fungible, have a traceable owner, and are tradable. The only difference is that we classify NFTs used to unlock access to further value held off the blockchain as Smart Collectibles.

It's important to note that not all NFTs are Smart Collectibles, but all NFTs have the potential to be classified as Smart Collectibles. If, at any time, the owner of an NFT (or anyone else) decides to link access to additional unique value to the ownership of that NFT, it can become a Smart Collectible.

Many NFTs currently sold do not offer access to unique value off the blockchain - they are not Smart Collectibles - but this may not be true in the future. It is likely that as the adoption of Smart Collectibles continues, more NFT creators and owners will want to add additional value.

\footnotetext{
19 thereum, EIP-721, https://github.com/ethereum/EIPs/blob/master/EIPS/eip-721.md

${ }^{20}$ Non-fungible tokens are not interchangeable, they have unique properties that set them apart from other nonfungible tokens. This is achieved by giving each token a unique tokenId.

${ }^{21}$ A decentralized application, or Dapp, is a program (such as a website or app) that runs on a distributed network.

${ }^{22}$ Ethereum, ERC-1155 Reference Implementation, https://github.com/enjin/erc-1155
} 


\subsection{Are Bitcoins Smart Collectibles?}

Fungible tokens, such as Bitcoins, are not Smart Collectibles. They hold value that can be realized by exchanging them for goods or services or transferred by exchanging them to fiat or another cryptocurrency. However, it is conceivable that these fungible tokens can act similarly. For example, a company might offer high net-worth Bitcoin holders certain advantages, such as access to a private members lounge or other benefits. In this scenario, Bitcoin acts as a smart collectible by unlocking access to value simply by being held.

\section{Looking to the Future: Smart Collectibles, Web 3.0, and the Metaverse}

The ability to provide access to almost any physical or digital value means Smart Collectibles have many interesting use cases, but it's the larger implications of this that are really exciting.

The functionality offered by Smart Collectibles is a key driver behind Web $3.0^{23}$, the third generation of the internet. Building on the foundations laid by Web $1.0^{24}$ and Web 2.025, Web 3.0 aims to deliver a more open internet, using technologies such as blockchain and AI to put people back in control of their data ${ }^{26}$.

Smart Collectibles will enable this by allowing users to control how the content they generate is used and to sell or trade it using a trustless system ${ }^{27}$ that does not rely on third parties, such as Facebook or Google. This also has the benefit that individuals will be able to transact anonymously, something that is impossible with Web 2.0 solutions.

In the future, specific accesses provided by a Smart Collectible will themselves be able to be tokenized, enabling owners to sell, lend, or duplicate parts of a Smart Collectible that they own while maintaining control of the underlying asset. The technology to do this is not available yet, but is expected to be in the future ${ }^{28}$.

Additionally, NFTs and Smart Collectibles are also key to the creation of the Metaverse - a long-anticipated always-on shared digital universe where people can work, play, and hang out. In the Metaverse, people will be represented by their avatars and need to own things - digital clothes, items, and even virtual land - and that's where NFTs and Smart Collectibles come in.

In a digital world where you can look however you like, the digital items you hold will become a key way of expressing your identity. While we're not at the Metaverse yet, worlds such as Decentraland, a virtual world built on the blockchain, and Fortnite, with its events and IP crossovers, are moving in that direction. It is hoped that within the next few years, many of these disparate

\footnotetext{
${ }^{23}$ Packy McCormick, The Value Chain of the Open Metaverse, https://www.notboring.co/p/the-value-chain-of-t he-open-metaverse

${ }^{24}$ The first phase of the internet, Web 1.0, was built on a set of open protocols - HTTP, FTP, SMTP, and others that allowed for the exchange of information and files between servers and enabled the first websites and email. The internet was decentralized but lacked both functionality (key protocols such as search and payments were missing) and accessibility (significant technical skills were required to use it).

${ }^{25}$ Web 2.0 built on Web 1.0 to provide the functionality and accessibility that the first phase lacked. Popular platforms like Facebook, Chrome, WhatsApp, Slack, YouTube, and Gmail have enabled billions of people to use the internet to search, chat, and buy \& sell online with little or no technical knowledge.

${ }^{26}$ Piers Kicks, Into The Void: Where Crypto Meets The Metaverse, https://metaversed.net/Into-The-Void-Whe re-Crypto-Meets-The-Metaverse-263f1ff8c13c455ea472f5689e01acaa

${ }^{27} \mathrm{~A}$ trustless system distributes trust between many participants, removing the need for a trusted third-party. This enables two people to enter into a transaction together even if they do not know and trust each other; the decentralized network ensures the transaction proceeds as agreed.

${ }^{28}$ DeStation is currently producing technology to solve this problem. Visit www.destation.org for updates.
} 
online worlds will join up into one Metaverse, which people will be able to travel across freely.

\section{Conclusion}

The possibilities offered by Smart Collectibles are nearly endless, but to see these become a reality, we need to see improvements in three areas:

Innovation - Businesses, communities, and creators must innovate in their use of NFTs, stacking value to create true Smart Collectibles. This functionality is available already, but is so far under-utilized. With the NFT ecosystem still at an early stage, there is a massive opportunity for businesses, communities, and creators to claim the advantage of being among the first to realize the true potential of this technology.

Participation - To encourage innovation, users must reward projects that seek to deliver more value than just metadata. Look out for creators, communities, and businesses that are stacking value and support them by purchasing their NFTs in favor of those who provide little value.

Interoperability - To achieve the true potential of the Metaverse, there must also be a solution for building interoperability between the multiple platforms that currently allow for the creation of Smart Collectibles, so that users can seamlessly transfer NFTs anywhere, providing vital liquidity for the system.

Regardless of whether you are a user, creator, or business, this is an exciting time to be learning about NFTs and Smart Collectibles. Below, we've provided several further reading suggestions that you may wish to check out.

\section{Acknowledgments}

Many thanks to all of the proofreaders who have helped get this into a vaguely presentable shape. In particular, Andrew Steinwold, Andrey Shirben, Benny Giang, David Hong, Ju Chun Ko, Kunal Modi, Lee Hsuan, Mateen Soudagar, Nagu Thogiti, Nick Rose, Nicholas Gregory, Yifat Shirben, and Zorayr Khalapyan. Thanks to all the people who have contributed ideas or the beginnings thereof, James Evans, Matthew Liu and Piers Kicks deserve especial mention. And thanks to everyone else for their help along the way. All errors are my own. ${ }^{29}$

${ }^{29}$ (C) 2021. This work is licensed under a CC BY 4.0; This license allows reusers to distribute, remix, adapt, and build upon the material in any medium or format, so long as attribution is given to the creator. The license allows for commercial use. https://creativecommons.org/licenses/by/4.0/ 


\section{Further Reading}

- Ethereum.org, Non-fungible Tokens (NFT), https://ethereum.org/en/nft/

- Ethereum, EIP-721, https://github.com/ethereum/EIPs/blob/master/EIPS/eip-721.md

- Ethereum, ERC-1155 Reference Implementation, https://github.com/enjin/erc-1155

- Satoshi Nakamoto, Bitcoin: A Peer-to-Peer Electronic Cash System, https://bitcoin.org/bit coin.pdf

- Nick Szabo, Smart Contracts: Building Blocks for Digital Markets, https://www.fon.hum.uv a.nl/rob/Courses/InformationInSpeech/CDROM/Literature/LOTwinterschool2006/szabo.be st.vwh.net/smart_contracts_2.html

- Packy McCormick, The Value Chain of the Open Metaverse, https://www.notboring.co/p/t he-value-chain-of-the-open-metaverse

- Piers Kicks, Into The Void: Where Crypto Meets The Metaverse, https://metaversed.net/I nto-The-Void-Where-Crypto-Meets-The-Metaverse-263f1ff8c13c455ea472f5689e01acaa

- Shermin Voshmgir, Tokenized Networks: Web3, the Stateful Web, https://blockchainhub.ne t/web3-decentralized-web/

- Edoardo Talamo \& Alma Pennacchi, IdToken: the new decentralized approach to digital identity, https://dblp.org/rec/conf/openidentity/TalamoP20 